\title{
Erratum
}

\section{Familial Risk of Type I diabetes in European Children}

\author{
The Eurodiab Ace Study Group and The Eurodiab Ace Substudy 2 Study Group \\ Diabetologia (1998) 41: 1151-1156 \\ The participating investigators and institutions are listed in the Appendix \\ Received: 16 February 1998 and in revised from: 4 May 1998 \\ Corresponding author: G. Soltész, Department of Paediatrics, University Medical School of Pécs, 7623 Pécs, József Attila u. 7., \\ Hungary
}

\section{Appendix}

\section{The Eurodiab Ace Substudy 2 Study Group}

Study co-ordinators: Gisela Dahlquist, Department of Paediatrics, Umea University, Umea, Sweden

Chris Patterson, Department of Epidemiology and Public Health, Queen' University Belfast, Northern Ireland, UK

Gyula Soltész, Department of Paediatrics, University of Pécs, Hungary

Study centre leaders:

Austria: Edith Schober and Ursula Schneider, Department of Paediatrics, University of Vienna, Vienna, Austria.

Bulgaria (western): Svetla Goranova, Mirela Ivanova, and Denka Stojanova, Children's Diabetic Centre, Sofia, Bulgaria.

Lithuania: Brone Urbonaite and Auste PundziuteLyczka, Institute of Endocrinology, Kaunas Medical Academy, Kaunas, Lithuania.

Latvia: Girts Brigis and Ilona Robeza, Department of Public Health and Epidemiology, Medical Academy of Latvia, Riga, Latvia.

Luxemburg: Carine de Beaufort, Clinique Pédiatrique, Luxemburg.
Romania: Anne-Marie Konnerth and Constantin Ionescu-Tirgoviste, Department of Nutrition and Metabolic Disease, University of Bucharest, Romania.

United Kingdom (Leeds): Patricia McKinney, Institute of Epidemiology and Health Services Research, University of Leeds, Leeds, UK.

United Kingdom (Northern Ireland): Chris Patterson and Ruth Greenlees, Department of Epidemiology and Public Health, Queens University of Belfast and David Hadden, Metabolic Unit, Royal Victoria Hospital, Belfast, UK.

Responsible for data processing and analysis:

Chris Patterson, Department of Epidemiology and Public Health, Queens University of Belfast, Belfast, UK.

Responsible for preparation of this manuscript:

Gisela Dahlquist, Department of Paediatrics, Umea University, Umea, Sweden

Chris Patterson, Department of Epidemiology and Public Health, University of Belfast, Northern Ireland, UK.

Gyula Soltész, Department of Paediatrics, University of Pécs, Hungary. 